\title{
Case Report: Severe COVID-19 and Dengue in an Indonesian Infant
}

\author{
Anggraini Alam, ${ }^{1 \star}$ Sri Sudarwati, ${ }^{1,2}$ Dzulfikar Djalil Lukmanul Hakim, ${ }^{1,2}$ and Sally Mahdiani ${ }^{2,3}$ \\ ${ }^{1}$ Department of Child Health Hasan Sadikin General Hospital, Faculty of Medicine Universitas Padjadjaran, Bandung, Indonesia; ${ }^{2}$ Limijati \\ Hospital, Bandung, Indonesia; ${ }^{3}$ Department of Ear Nose and Throat Hasan Sadikin General Hospital, Faculty of Medicine Universitas Padjadjaran, \\ Bandung, Indonesia
}

\begin{abstract}
We report a case of a 10-month infant with dual severe infection of COVID-19 and dengue fever who was admitted to the hospital with an influenza-like illness. The patient experienced severe conditions of COVID-19 and dengue fever with shock followed by disseminated intravascular coagulation. The standard of COVID-19 care was given coupled with fluid resuscitation and blood transfusion. The pitfalls of this case are how to differentiate the clinical manifestation of dengue fever in a patient with confirmed COVID-19; the difficulty to monitor the dengue course of illness of the patient in the COVID-19 isolation room; and to differentiate the severe dengue from the multisystem inflammatory syndrome-C when the patient was in critical condition. The infant recovered without sequale, but the management of new probable cases must be improved more thoroughly, especially during dengue peak period in tropical and developing countries such as Indonesia.
\end{abstract}

\section{INTRODUCTION}

There were 671,778 confirmed cases of COVID-19 and 20,085 deaths in Indonesia in December $21 .{ }^{1}$ Simultaneously, dengue fever has also increased, with 95,971 cases and 663 deaths until December 2020. ${ }^{2}$ Some studies reported fever as the most common symptom of children with confirmed COVID-19 as well as dengue, and the clinical manifestations of both are difficult to be distinguished, ${ }^{3-9}$ especially for those who lived within dengue-endemic area, such as Indonesia. Thus, the possibility of dual infection between COVID-19 and dengue infection should be considered in feverish children in dengue-endemic areas during the COVID-19 pandemic.

Both COVID-19 and dengue had their antibody-dependent enhancement (ADE) mechanism that causes shock, coagulopathy, and increase of inflammatory markers. ${ }^{10,11}$ In a severe case, we need to analyze whether shock happened because of dengue or COVID-19 multisystem inflammatory syndromeC (MIS-C). It is important to identify which disease has caused shock to determine appropriate management.

\section{CASE PRESENTATION}

This is our first case of severe dengue in an infant with COVID-19. A 10-month-old girl, $7 \mathrm{~kg}$, presented with fever, cough, and vomiting. She had no contact history with confirmed cases of COVID-19, but she resided in Bandung, a city with high local transmission of COVID-19. Her father works as a motorcycle taxi rider, and her mother trades in her stall. No influenza-like history in the family or neighbors. Before admission, she was treated with paracetamol for 2 days, but the fever still persisted. On the fourth day, the symptoms were accompanied by dyspnea.

On admission, the patient was alert, with a temperature of $38.5^{\circ} \mathrm{C}$, respiratory rate of $60 \mathrm{rpm}$, pulse rate of $140 \mathrm{bpm}$, and $\mathrm{SpO}_{2} 88 \%$ in room air. Chest indrawing and crackles were found. The blood count results are as follows: lymphopenia with a total lymphocyte count (TLC) of 796.8 , neutrophil-

\footnotetext{
* Address correspondence to Anggraini Alam, Pediatric Infectious and Tropical Disease, Department of Child Health Hasan Sadikin General Hospital, Faculty of Medicine Universitas Padjadjaran, Jl. Pasteur No. 38, Bandung 40161, Indonesia. E-mail: anggialam@yahoo.co.id
}

lymphocyte ratio 4.8 , and increased C-reactive protein (CRP) to $29 \mathrm{mg} / \mathrm{dL}$. Chest X-ray (CXR) showed multiple small patches at peripheral and small nodules on perihelial and pericardial sites, which indicate interstitial processes on both the lungs (Figure 1A). She was suspected of having COVID-19 infection and then admitted to the isolation room. Binasal cannula oxygen therapy was given from day 1 to day 7 , and also IV fluid (normal saline), paracetamol, antibiotic, and vitamin.

Considering acute fever and her living environment in dengue-endemic area, nonstructural-1 (NS-1) dengue was also checked, and the result was positive. On day 5 of hospitalization, she looked drowsy, whereas the fever's subset, heart rate increased, and dark color of gastric residual occurred with cold extremities and liver enlargement. Shock, gastric bleeding, and melena were also observed. The platelet count dropped to $25,000 / \mu \mathrm{L}$ with coagulopathy (prothrombin time 13.2", activated partial thromboplastin time 44.9", and d-dimer 1,373.34) and increased aspartate and alanine aminotransferase $(1,344 \mathrm{~g} / \mathrm{dL}$ and $618 \mathrm{~g} / \mathrm{dL})$, concurrent with hemoconcentration (the lowest was $29.5 \%$ and the highest was $42.5 \%$ ) and pleural effusion (Figure 1B).

She was diagnosed with severe dengue (shock and disseminated intravascular coagulation [DIC]). Her condition was improved after fluid resuscitation, oxygenation (simple mask $5 \mathrm{~mL} /$ minute), and blood transfusion (platelet and fresh frozen plasma) were given. On day 7 , the convalescent rash appeared. However, no fever or shortness of breath was observed, so no oxygen was required. Cough was also decreased gradually at that time. On day 10 , the result of the reverse transcriptase polymerase chain reaction (RT-PCR) SARS-CoV-2 test was positive with cycle threshold (Ct) of 37 , and she was transferred to a referral hospital for COVID-19.

The RT-PCR SARS-CoV-2 test was actually performed on day 1 of her hospital admission, but the test result was delayed. After the patient was transferred, RT-PCR was performed again on day 11 and day 15, and the result was negative. Only nasopharyngeal (NP) specimens were collected for COVID-19 examination.

She was discharged after 16 days of hospitalization, with final diagnoses of severe COVID-19 and severe dengue. All the hospital expenses were covered by the national insurance. 
A

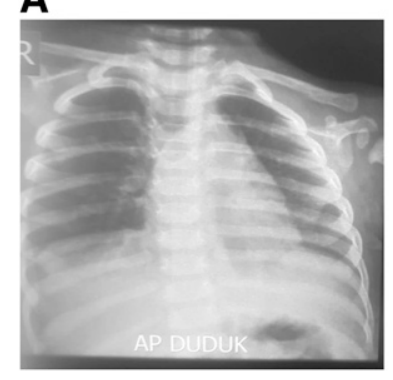

Day-1: bronchopneumonia
B

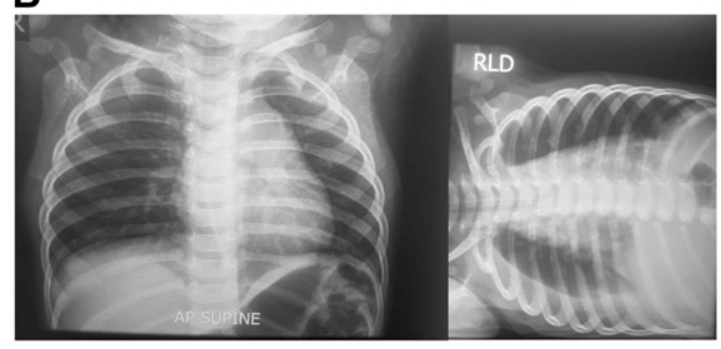

Day-5: Right pleura effusion

FIGURE 1. (A) Day 1: bronchopneumonia. (B). Day 5: right pleura effusion.

A thorough contact tracing process within her core family was performed; and their RT-PCR test was negative for COVID-19.

\section{DISCUSSION}

To our knowledge, this is the first reported case of severe COVID-19 and severe dengue in an infant. Therefore, we need to confirm the clinical manifestations of both diseases because the case has been rarely reported.

COVID-19 is diagnosed based on epidemiological history, clinical manifestations, and laboratory examination, and confirmed by RT-PCR examination. ${ }^{4-8}$ In our case, the patient lived in a city with local transmission of COVID-19 with a positivity rate of $8.7 \%$ in July $2020 .^{12}$ Taking available evidences into account, they indicate that infant was most likely to have COVID-19. ${ }^{6}$ We presumed that the patient might have been infected by the virus via community transmission because the mother always brought her to the trading stall. Even so, the tracing performed on her parents showed negative results of COVID-19.

The common manifestations of COVID-19 are fever, cough, and shortness of breath. The patient showed all those symptoms, accompanied by vomiting, which is often found in children with COVID-19 (Table 1), and also lymphopenia, low TLC, and NLR > 3.31 (Table 2). ${ }^{4-8,13}$ When admitted to the hospital, the chest CXR found an abnormality that is generally caused by the viral infection, so the radiology expertise's interpretation was viral bronchopneumonia. Computed tomography scan was not performed because of the unavailability of the resources. In a study of 7,780 pediatric patients, $76.4 \%$ and $81.1 \%$ had CXR and CT abnormality, respectively; CXR appearance of viral infection was observed in 105 of $501 .^{14}$ Based on these various findings, we concluded the patient contracted COVID-19.

COVID-19 was confirmed according to the Ct values ascertained by the RT-PCR assay, with a Ct value $<40$ being considered positive. ${ }^{14,15}$ Apparently, the patient had developed severe condition which was not correlated with her Ct value (37). This study fails to find a significant relationship between $\mathrm{Ct}$ value and severity of illness. ${ }^{16}$

Because dengue should be considered in feverish children lived in an endemic region, ${ }^{17} \mathrm{NS}-1$ test should be performed in the early day of fever to confirm dengue infection. ${ }^{18}$ The IgM and IgG of anti-dengue seemed not reliable as a confirmatory test for dengue because it could be false positive when the patient also contracted COVID-19. ${ }^{19}$

TABLE 1

Signs and symptoms

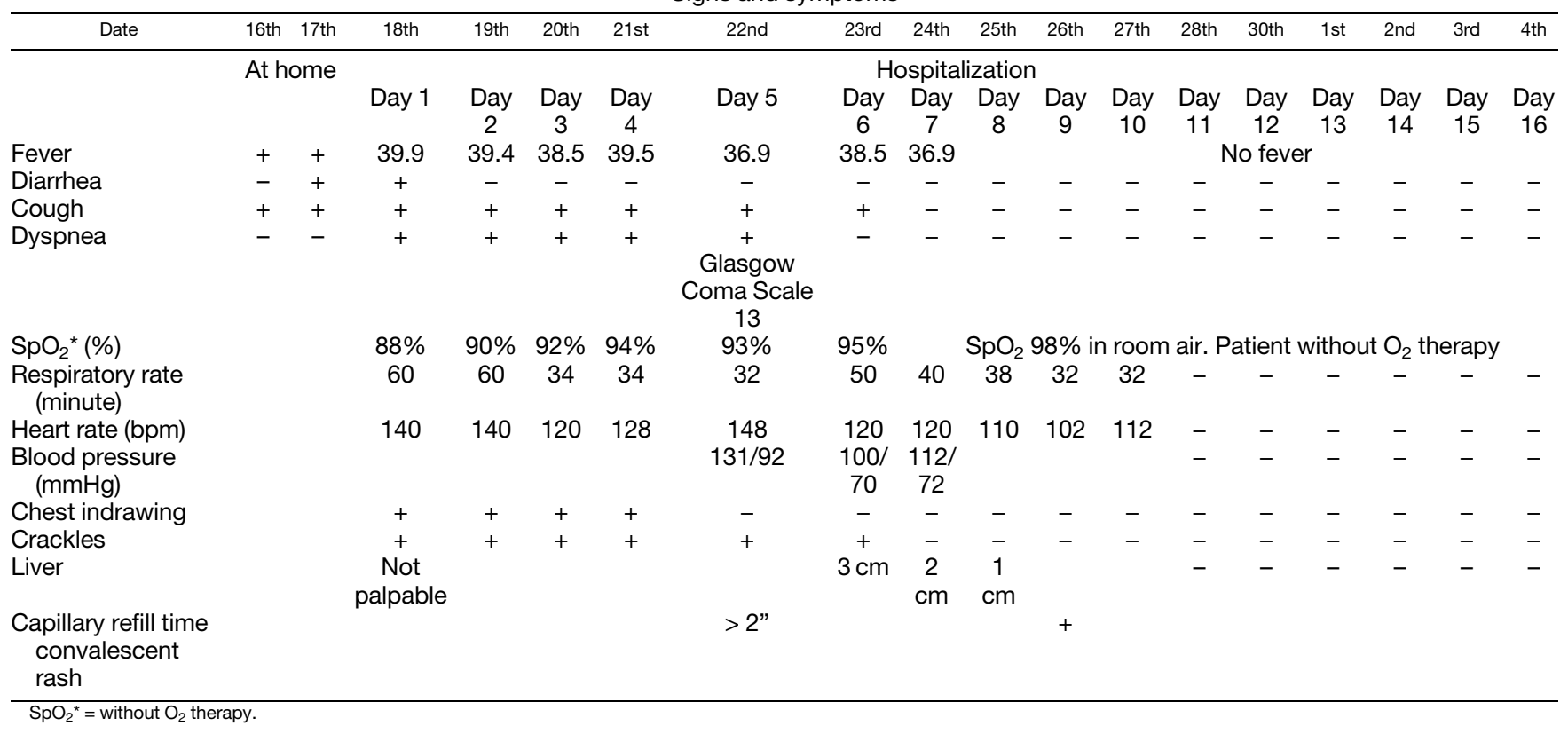


TABLE 2

Laboratory results

\begin{tabular}{|c|c|c|c|c|c|c|c|c|c|c|c|}
\hline Hospitalization & \multicolumn{2}{|c|}{ Day 1} & Day 2 & Day 3 & \multicolumn{2}{|c|}{ Day 4} & \multicolumn{2}{|c|}{ Day 5} & Day 6 & Day 7 & Day 8 \\
\hline Hemoglobin (g/dL) & \multicolumn{2}{|c|}{$11.5 \mathrm{~g} / \mathrm{dL}$} & 11.5 & 11.6 & \multicolumn{2}{|c|}{12} & \multicolumn{2}{|c|}{14.3} & 10.5 & 10.7 & 9.8 \\
\hline Hematocrit (\%) & \multicolumn{2}{|c|}{$33 \%$} & 34 & 34 & \multicolumn{2}{|c|}{37.5} & \multicolumn{2}{|c|}{42.5} & 30 & 33 & 30 \\
\hline Platelet $\left(\mathrm{mm}^{3}\right)$ & \multicolumn{2}{|c|}{$213,000 / \mathrm{mm}^{3}$} & 169,000 & 120,000 & \multicolumn{2}{|c|}{97,000} & \multicolumn{2}{|c|}{25,000} & 57,000 & 52,000 & 75,000 \\
\hline Leukocyte $\left(\mathrm{mm}^{3}\right)$ & \multicolumn{2}{|c|}{$4,980 / \mathrm{mm}^{3}$} & 3,360 & 3,240 & \multicolumn{2}{|c|}{3,470} & \multicolumn{2}{|c|}{4,050} & 11,020 & 8,920 & 10,820 \\
\hline Eosinophil (\%) & \multicolumn{2}{|c|}{1} & - & - & \multicolumn{2}{|c|}{-} & \multicolumn{2}{|c|}{ PT 13.7} & - & - & - \\
\hline Basophil (\%) & & & - & - & & & aPTा & 4.9 & - & - & - \\
\hline Neutrophil (\%) & & & - & - & & & d-Dimer 1, & 3.34 & - & - & - \\
\hline Lymphocyte (\%) & & & - & - & & & AST 1 & & - & - & - \\
\hline Monocyte (\%) & & & - & - & & & ALT 6 & & - & - & - \\
\hline TLC & & & - & - & & & $(-)$ & & - & - & - \\
\hline NLR & & & - & - & & & $(-)$ & & - & - & - \\
\hline CRP & & & - & - & & & $(-)$ & & - & - & $<5$ \\
\hline NS-1 & & & $(+)$ & & & & & & & & \\
\hline PCR COVID-19 & Nasopha & yeal swab & - & - & & & - & & - & - & - \\
\hline Hospitalization & Day 9 & Day 10 & Day 11 & Day 1 & & Day 13 & Day 14 & Day 15 & & & Day 17 \\
\hline Hemoglobin (g/dL) & 9. & 9.8 & - & - & & - & - & - & & & 10.3 \\
\hline Hematocrit (\%) & 29 & 30 & - & - & & - & - & - & & & 30.4 \\
\hline Platelet $\left(\mathrm{mm}^{3}\right)$ & 142,000 & 173,000 & - & - & & - & - & - & & & 498,000 \\
\hline Leukocyte $\left(\mathrm{mm}^{3}\right)$ & 11,130 & 10,640 & - & - & & - & - & - & & & 8,350 \\
\hline Eosinophil (\%) & - & - & - & - & & - & - & - & & & 2 \\
\hline Basophil (\%) & - & - & - & - & & - & - & - & & & 0 \\
\hline Neutrophil (\%) & - & - & - & - & & - & - & - & & & 16 \\
\hline Lymphocyte (\%) & - & - & - & - & & - & - & - & & & 71 \\
\hline Monocyte (\%) & - & - & - & - & & - & - & - & & & 11 \\
\hline TLC & - & - & - & - & & - & - & - & & & $5,928.5$ \\
\hline NLR & - & - & - & - & & - & - & - & & & 0.23 \\
\hline CRP & - & - & - & - & & - & - & - & & & - \\
\hline PCR COVID-19 & - & $\begin{array}{l}\text { First result: } \\
\qquad(+) \text { positive }\end{array}$ & - & $\begin{array}{l}\text { Second } r \\
(-) \text { neg }\end{array}$ & & - & - & - & $\begin{array}{l}\text { Third re } \\
\text { (-) n }\end{array}$ & $\begin{array}{l}\text { It: } \\
\text { ative }\end{array}$ & - \\
\hline
\end{tabular}

Did the patient suffer from a severe dengue or severe form of COVID-19 in children as now named as a MIS-C? In severe cases, both dengue and COVID-19 patients may clinically deteriorate rapidly and develop hemodynamic compromise; when in MIS-C, a patient would have a fever, more prominent vomiting, and show significant lymphopenia and increased of D-dimer. ${ }^{14}$ Apparently, in our case, the patient had a fever, acute vomiting, lymphopenia, rapidly decreased platelet, and elevated CRP and liver function enzyme level, followed by shock and DIC. But, however, she did not meet other MIS-C conditions such as mucocutaneous and cardiac involvement. ${ }^{20}$ Her condition was improved after having fluid resuscitation (Ringer's lactate and colloid) and blood transfusion (platelet and fresh frozen blood), without any treatment for MIS-C. ${ }^{21}$ Therefore, we concluded the shock manifestation was due to dengue which was also confirmed by NS-1 positive and signs of plasma leakage (the decreased hematocrits and pleural effusion is not a specific sign of COVID-19 or MIS-C). The patient had a convalescent rash as the hallmark of dengue infection in a recovery phase that convinced us that she had dengue. ${ }^{9,17,22}$ What we really need to put attention to is that lymphocytosis and monocytosis pattern in dengue may be changed by the presence of COVID-19.

The deterioration of the patient's condition might be influenced by her age. Even though the infant might have received dengue antibodies vertically, the preexisting antibodies decreased with age, followed by the dengue virus, as a primary infection, entered ADE, and exacerbated the dengue symptoms. ${ }^{10}$ On the other hand, ADE mechanism in the MIS-C of COVID-19 becomes an interesting subject that needs to be studied further, because the exact MIS-C mechanism is not yet known, ${ }^{11}$ and potential interaction of pathophysiology between COVID-19 and dengue infection is still not certain.
COVID-19 may get worse if the infection is concurrent with dengue or vice versa, especially in infants. Consequently, continuous hemodynamic monitoring and management in dual infection case remain crucial.

Moreover, isolating a patient with COVID-19 and dengue dual infection can be tough. In dengue wards, patient should be monitored closely, but in this case, we missed to identify the patient's shock condition because she was treated in COVID-19 isolation room with only limited visits allowed..$^{9,22}$

Antiviral for children infected by COVID-19 remains challenging in resource-limited countries. We had to confirm first that the patient had been infected by COVID-19 before providing the appropriate treatments. In this case, the confirmation result of COVID-19 laboratory examination was delayed until 10 days. By that time, the patient's condition had improved already, so she no longer needed any COVID-19 treatments. ${ }^{23,24}$

After 16 days of hospitalization, the COVID-19 PCR test was negative. She was discharged with a complete clinical improvement, ${ }^{16}$ and survived without any sequelae. Figure 2 showed the course of her illness. Because COVID-19 has longer incubation time than dengue, so in this case, COVID-19 seemed to occur first followed by dengue infection.

We concluded that during the COVID-19 pandemic, coinfection of COVID-19 and dengue should be considered in feverish children living in endemic tropical region of dengue, especially on dengue peak case period (by the end of rainfall season). More importantly, in developing countries with health facility constrained, we need to pay a very careful attention in treating coinfection cases: 1) The pitfall in identifying the poor signs of peripheral perfusion, as early sign of shock during dengue critical phase, when a patient is treated in COVID-19 isolation room. 2) Estimation of "the period of critical state" in 


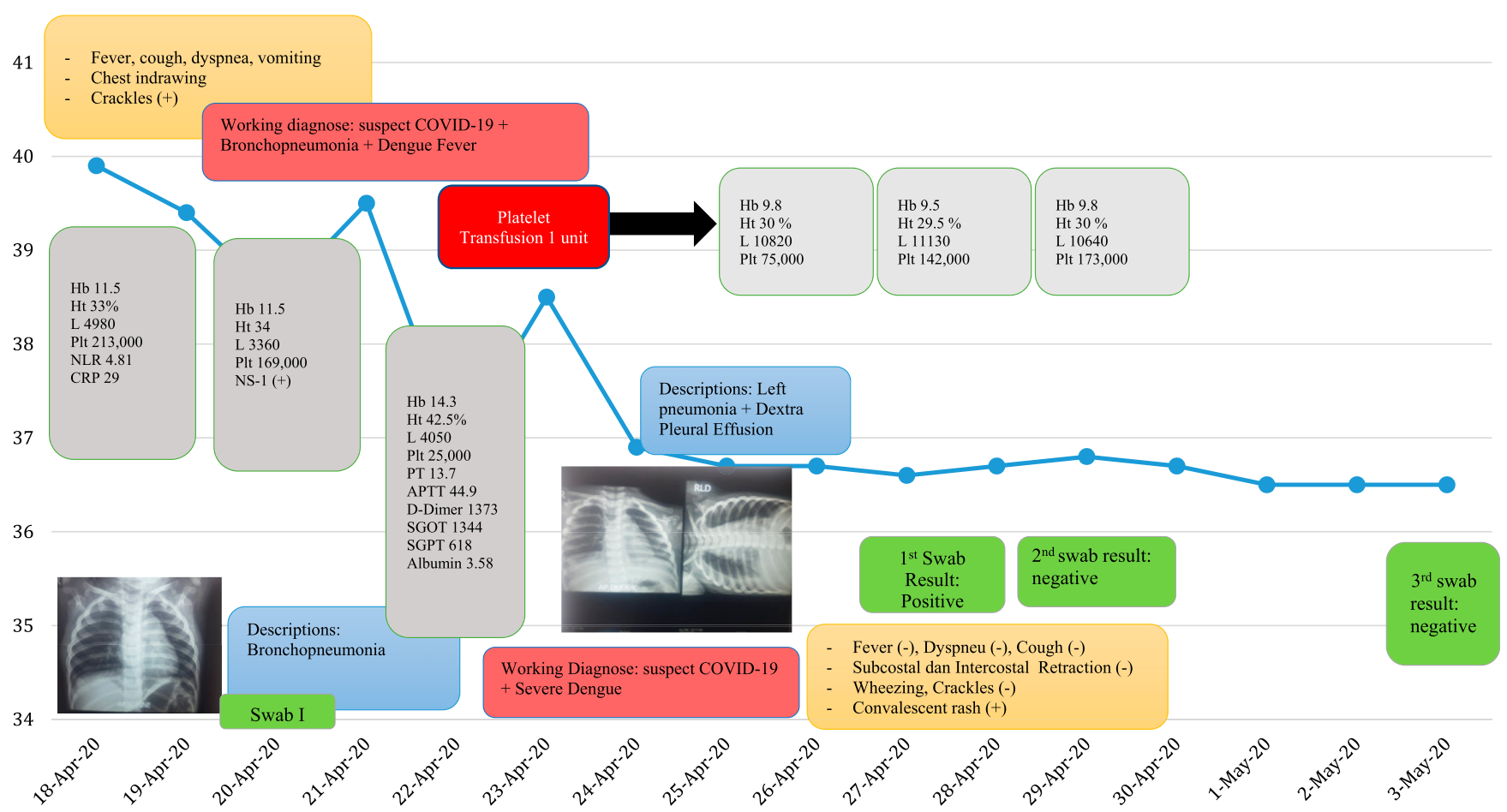

FIGURE 2. Course of illness. This figure appears in color at www.ajtmh.org.

dengue and COVID-19, especially the MIS-C, is crucial to determine early intervention and prevent deaths.

Received September 23, 2020. Accepted for publication February 9, 2021.

Published online February 24, 2021.

Acknowledgments: We thank the emerging and reemerging team of Hasan Sadikin General Hospital, especially pediatric COVID-19 team, and healthcare workers of Limijati Hospital for supporting care of the patient. Publication charges for this article were waived due to the ongoing pandemic of COVID-19.

Financial support: The author(s) received no additional financial support but self-sufficiency for this work.

Disclosure: The authors declare that this manuscript is original, has not been published before, and is not currently being considered for publication. Informed consent was obtained from the patient for management of the patient.

Authors' addresses: Anggraini Alam, Sudarwati, and Dzulfikar Djalil Lukmanul Hakim, Department of Child Health Hasan Sadikin General Hospital, Faculty of Medicine Universitas Padjadjaran, Bandung, Indonesia, E-mails: anggialam@yahoo.co.id, srisumarwan@yahoo.com, and dzulfikar_dlh@yahoo.com. Sally Mahdiani, Department of Ear Nose and Throat Hasan Sadikin General Hospital, Faculty of Medicine Universitas Padjadjaran, Bandung, Indonesia, and Limijati Hospital, Bandung, Indonesia, E-mail: sally.mahdiani@gmail.com.

This is an open-access article distributed under the terms of the Creative Commons Attribution (CC-BY) License, which permits unrestricted use, distribution, and reproduction in any medium, provided the original author and source are credited.

\section{REFERENCES}

1. Satgas Penanganan COVID-19 Indonesia, 2020. Situasi Virus COVID-19 di Indonesia. Available at: https://covid19.go.id. Accessed February 16, 2021.

2. Indonesia Ministry of Health, 2020. Dengue Data JanuaryDecember 2020. Surveillance of Ministry of Health Indonesia. Jakarta, Indonesia: Kementrian Kesehatan.
3. CDC COVID-19 Response Team, 2020. Coronavirus disease 2019 in children-United States, February 12-April 2, 2020. Morbidity Mortality Weekly Rep 69: 422.

4. Dong Y, Mo X, Hu Y, Qi X, Jiang F, Jiang Z, Tong S, 2020. Epidemiology of COVID-19 among children in China. Pediatrics 145: e20200702.

5. Lu X et al., 2020. SARS-CoV-2 infection in children. New Engl J Med 382: 1663-1665.

6. Parri N, Lenge M, Buonsenso D, 2020. Children with COVID-19 in pediatric emergency departments in Italy. New Engl J Med 383: 187-190.

7. Shen K et al., 2020. Diagnosis, treatment, and prevention of 2019 novel coronavirus infection in children: experts' consensus statement. World J Pediatr 16: 223-231.

8. Wu D, Wu T, Liu Q, Yang Z, 2020. The SARS-CoV-2 outbreak: what we know. Int $J$ Infect Dis 94: 44-48.

9. World Health Organization, 2009. Dengue: Guidelines for Diagnosis, Treatment, Prevention and Control. Geneva, Switzerland: World Health Organization, 1-147.

10. Jain A, Chaturvedi UC, 2010. Dengue in infants: an overview. FEMS Immunol Med Microbiol 59: 119-130.

11. Jin Y, Yang H, Ji W, Wu W, Chen S, Zhang W, Duan G, 2020. Virology, epidemiology, pathogenesis, and control of COVID19. Viruses 12: 372.

12. The Information and Coordination Centre for Disease and Disaster inWest Java. Available at: https://pikobar.jabarprov.go.id. Accessed July 12, 2020.

13. Hoang A, Chorath K, Moreira A, Evans M, Burmeister-Morton F, Burmeister F, Naqvi R, Petershack M, Moreira A, 2020. COVID-19 in 7780 pediatric patients: a systematic review. EClinicalMedicine 24: 100433.

14. Zou L et al., 2020. SARS-CoV-2 viral load in upper respiratory specimens of infected patients. New Engl J Med 382: 1177-1179.

15. Xu T et al., 2020. Clinical features and dynamics of viral load in imported and non-imported patients with COVID-19. Int J Infect Dis 94: 68-71.

16. Hu X et al., 2020. Factors associated with negative conversion of viral RNA in patients hospitalized with COVID-19. Sci Total Environ 728: 138812.

17. Utama IMS et al., 2019. Dengue viral infection in Indonesia: epidemiology, diagnostic challenges, and mutations from an 
observational cohort study. PLoS Negl Trop Dis 13: e0007785.

18. Lorenz C, Azevedo TS, Chiaravalloti-Neto F, 2020. COVID-19 and dengue fever: a dangerous combination for the health system in Brazil. Travel Med Infect Dis 35: 101659.

19. Yan G et al., 2020. Covert COVID-19 and false-positive dengue serology in Singapore. Lancet Infect Dis 20: 536.

20. World Health Organization, 2020. Multisystem Inflammatory Syndrome in Children and Adolescents Temporally Related to COVID-19. Available at: https://www.who.int/news-room/ commentaries/detail/multisystem-inflammatory-syndrome-inchildren-and-adolescents-with-covid-19. Accessed September 14, 2020.
21. Riphagen S, Gomez X, Gonzalez-Martinez C, Wilkinson N, Theocharis $P, 2020$. Hyperinflammatory shock in children during COVID-19 pandemic. Lancet 395: 1607-1608.

22. World Health Organization, 2012. Handbook for Clinical Management of Dengue WHO and Special Programme for Research and Training in Tropical Diseases (TDR). Geneva, Switzerland: World Health Organization.

23. Zimmermann $P$, Curtis $N, 2020$. Coronavirus infections in children including COVID-19: an overview of the epidemiology, clinical features, diagnosis, treatment and prevention options in children. Pediatr Infect Dis J 39: 355-368.

24. Wang Y, Zhu LQ, 2020. Pharmaceutical care recommendations for antiviral treatments in children with coronavirus disease 2019. World J Pediatr 16: 271-274. 